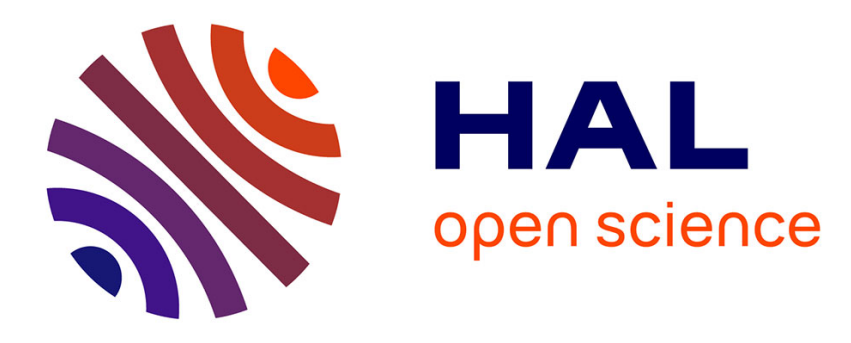

\title{
Statistical approach for radial fingering in a Hele Shaw cell
}

\author{
J. Geminard, P. Pelce
}

\section{To cite this version:}

J. Geminard, P. Pelce. Statistical approach for radial fingering in a Hele Shaw cell. Journal de Physique II, 1992, 2 (11), pp.1931-1940. 10.1051/jp2:1992244 ～jpa-00247779

\section{HAL Id: jpa-00247779 https://hal.science/jpa-00247779}

Submitted on 1 Jan 1992

HAL is a multi-disciplinary open access archive for the deposit and dissemination of scientific research documents, whether they are published or not. The documents may come from teaching and research institutions in France or abroad, or from public or private research centers.
L'archive ouverte pluridisciplinaire HAL, est destinée au dépôt et à la diffusion de documents scientifiques de niveau recherche, publiés ou non, émanant des établissements d'enseignement et de recherche français ou étrangers, des laboratoires publics ou privés. 


\title{
Statistical approach for radial fingering in a Hele Shaw cell
}

\author{
J. C. Geminard $\left({ }^{1}\right)$ and P. Pelce $\left({ }^{2}\right)$ \\ (1) Laboratoire de Physique de l'E.N.S. Lyon, 46 Allée d'Italie, 69364 Lyon Cedex 07, France \\ (2) Laboratoire de Recherche en Combustion, Université de Provence, St Jérôme, 13397 \\ Marseille Cedex 13, France
}

(Received 24 July 1992, accepted 3 August 1992)

\begin{abstract}
We have used a statistical method to describe an unsteady radial fingering pattern. We determine a complete set of equations for the growth of the microstructure envelope which couples envelope radius, individual growth rate of fingers and the distribution function in the cell size space. In a preliminary approach, we neglect the important screening effect which may be responsible for the commonly observed fractal structures. In that case, the structure is compact, i.e. the relative width of fingers of order unity. The self-similar regime for which the time dependence of the envelope radius is in $t^{1 / 2}$ is then still a solution. The corresponding distribution function in the $A$-space varies with $t^{1 / 4}$. Contrary to regular structures for which any wavelength and thus any growth velocity of the envelope can be a solution, the self-similar solution found here determines unambiguously the growth velocity of the radial pattern. This velocity is a function of the amplitude of the noise present in the Hele Shaw cell. Numerical integration of the equations is performed and the time evolution of the distribution function starting from various initial conditions analysed.
\end{abstract}

\section{Introduction.}

When a viscous fluid displaces another more viscous fluid in a Hele Shaw cell, long fingers of the displacing fluid penetrate into the displaced fluid. When the displacing fluid is introduced at the center of the cell at constant volume flow rate $Q b$, where $b$ is the thickness of the cell, the fingers expand radially. Then, an unsteady interface develops [1] where two basic mechanisms take place : fingers frequently tip-split when they reach a critical size, and some of them are screened by the faster ones leaving large holes in the final pattern. This is a prototype of an unsteady microstructure with well defined statistical properties.

From experimental observations, the unsteady microstructure can be decomposed in an assembly of elementary cells growing in size because of the broadening effect associated with the increase of the mean radius of the envelope. They tip-split periodically when a critical size is reached and eventually disappear because of the screening effect. Because of our lack of knowledge of the initial conditions it is natural to describe the microstructure by a distribution function from which all usual statistical quantities can be determined. The natural degree of freedom associated to a unit cell is its size $A$ since it has been shown (see for instance Ref. [2]) that as far as the width of a channel in which a finger develops is given, an asymptotic steady state is reached in a unique fashion. It follows that the distribution function describing the 
statistical properties of the system will be defined in the $\Lambda$ space (an analogous method was used in eutectic solidification [3]).

The development of the method used here is inspired by the analysis of Lifshitz and Slyozov [4] of the early stage of the coalescence of grains in a supersaturated solution. In that case the system is described by a distribution function in the grain radius space. The grains are assumed to grow without direct interaction. But the total quantity of impurities released by the grains when they grow decreases the supersaturation and slows the growth of all the grains. Thus there is a coupling between the supersaturation, the individual growth rate of each grain and the distribution function in the grain radius space.

Here, the system is described by a distribution function in the cell size space. The elementary cells are assumed to grow without direct interaction. Here, the time-dependent quantity playing the role of the supersaturation in the former problem is the radius $R(t)$ of the envelope. Thus there is a coupling between, effective interface radius, individual growth rate for a unit cell and the distribution function in the $A$-space.

To proceed further, we neglect the important mechanism of cell elimination which may be responsible for the commonly observed fractal structures. This allows us to understand how an assembly of cells growing individually with different relative widths, and thus different velocities, behave collectively to determine an effective circular microstructure of radius $R(t)$. With this assumption, we are able to determine a complete set of equations for this problem which couple the effective germ radius $R(t)$, and the distribution function in the $\Lambda$ space. Since the relative proportion of the displacing fluid remains of the same order during the growth, one finds again a self-similar solution, for which $R(t)$ evolves like $t^{1 / 2}$ The corresponding distribution function in the $A$-space, which scales like $t^{1 / 4}$ can be determined analytically. In contrast with periodic cellular interfaces, for which any wavelength and thus any growth velocity is allowed, the self-similar solution determined here selects unambiguously the growth velocity of the microstructure.

\section{The model.}

The basic assumption of the analysis is that the interface between the two fluids is considered as an assembly of elementary units growing without direct interaction between them. The envelope of these units is assumed to be a circle of radius $R(t)$, large compared to the individual size $A$ occupied by each basic element. Each unit is a finger growing radially with velocity $v$ in a sector of angle $A / R$ (Fig. 1). From a geometrical consideration, the increase of the radius of the envelope leads to an increase of the size of each elementary unit [5]:

$$
\frac{\mathrm{d} \Lambda}{\mathrm{d} t}=\frac{\Lambda v}{R}
$$

where all possible tangential motion of an elementary cell is neglected.

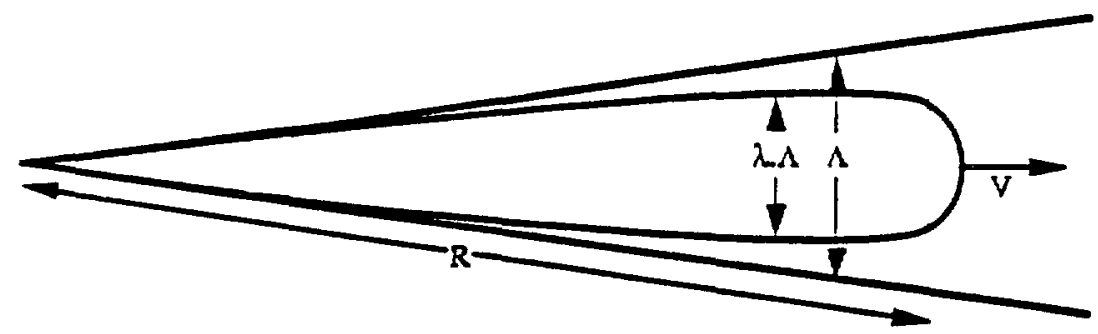

Fig. 1. - Sketch of an elementary cell growing in an angular sector of angle $A / R$. 
It is customary to introduce the relative proportion of displacing fluid inside a unit cell, $\lambda$. This factor appears as an eigenvalue of the free-boundary problem which determines the shape of the growing finger and is a function of the control parameters of the growth [6]. For instance, when the angle of the sector is assumed small, which is the case when the radius of the envelope becomes large, the cell shape is a Saffman-Taylor finger [6] of relative width $\lambda(C)$, a function of the parameter $C=3 \mu \Lambda^{2} v / \sigma b^{2} \pi^{2}$. Here, $\sigma$ is the surface tension between the two fluids and $\mu$ the viscosity of the displaced fluid, assumed much larger than the viscosity of the displacing fluid. One can deduce the finger velocity from the mass conservation relation

$$
\lambda v=\frac{Q}{2 \pi R}
$$

where $Q / 2 \pi R$ is the velocity of the fluid just ahead of the fingers.

When the size of a sector enlarges because of the increase of the radius of the envelope, the corresponding finger splits generally in two parts that we assume of equal size. When one considers growth in a sector of finite angle, tip-splitting results [7] from a branch merger of two Vanden-Broeck [8] solutions. But when the radius of the envelope increases, the sector angle decreases, so that the usual cause of tip-splitting in rectilinear Hele-Shaw cells, i.e. the noise is responsible. The now well known corresponding instability criterium [2] is simply that the control parameter $C$ is larger than a critical value $C_{\max }$ which depends on the amplitude of the noise in the Hele Shaw cell. The tip-splitting operates at constant outer fluid velocity $Q / 2 \pi R$ so that a newly formed semi-cell is associated to a control parameter $C_{\min }$ satisfying $g\left(C_{\min }\right)=g\left(C_{\max }\right) / 4$, where $g(C)=\lambda(C) C$. Thus the domain of possible wave-length $A$ is the segment $\left[\Lambda_{\min }(t), \Lambda_{\max }(t)\right]$, where, from the expression of $C$ and equation (2), one obtains :

$$
A_{\max }(t)=\sqrt{g\left(C_{\max }\right) R(t) \frac{b}{\mathrm{Ca}}}
$$

where $\mathrm{Ca}$ is the capillary number $\mathrm{Ca}=\left(3 / 2 \pi^{3}\right) \mu Q / \sigma b$.

Let $n(A, t)$ be the wavelength distribution function, normalized so that

$$
2 \pi R(t)=\int_{\Lambda_{\min }(t)}^{\Lambda_{\max }(t)} A n(\Lambda, t) \mathrm{d} \Lambda .
$$

Hence, $n(\Lambda, t) \mathrm{d} \Lambda$ is the number of cells on the effective germ of size between $\Lambda$ and $\Lambda+\mathrm{d} \Lambda$. From equations (1) and (2), one obtains

$$
v_{\Lambda}=\frac{\mathrm{d} A}{\mathrm{~d} t}=\frac{Q b}{2 \pi \mathrm{Ca} \Lambda R(t)} g^{-1}\left(\frac{\Lambda^{2}}{b R(t)} \mathrm{Ca}\right)
$$

as the rate of motion in the $\Lambda$ space. Then, the continuity equation in that space reads

$$
\frac{\partial n}{\partial t}+\frac{\partial}{\partial \Lambda}\left(n v_{\Lambda}\right)=0
$$

with the boundary condition $\left(n v_{A}\right)\left(A_{\min }\right)=2\left(n v_{A}\right)\left(A_{\max }\right)$, which expresses the tip-splitting rule.

Here, we neglect the cell death process which occurs when one cell backs away from two neighbouring ones. This process is important since it mainly determines the large scale 
properties of the microstructure. However no way to take it into account has been found for the moment.

When the function $\lambda(C)$ is given, equations (4)-(6) form a complete set of equations for the distribution function $n$ and the radius of the envelope $R(t)$ as a function of $C_{\max }$.

It is interesting to notice that this problem is analogous to the well known problem of the early stage of coalescence of precipitated grains solved by Lifshitz and Slyozov [4]. In the latter case, the statistical variable is the radius a of a grain whose individual growth rate depends on the supersaturation $\Delta(t)$. This quantity is coupled to the statistical problem by the conservation of impurities. It is well known that in this case this coupling leads to an evolution law for the mean grain radius $\bar{a} \approx t^{1 / 3}$ and not $\bar{a} \approx t^{1 / 2}$ as for a growth at constant supersaturation.

For practical purposes, simply because the boundaries of the domain remain independent of time, it is more convenient to work in the $C$-than in the $A$-space, the relation between them being determined by equation (3). With this new space variable, equations (4)-(6) become :

$$
\begin{gathered}
2 \pi \sqrt{\frac{R(t)}{b} \mathrm{Ca}}=\int_{C_{\min }}^{C_{\max }} \sqrt{g(C)} n(C, t) \mathrm{d} C \\
g^{\prime}(C) \frac{\mathrm{d} C}{\mathrm{~d} t}=\frac{Q}{\pi R^{2}} C-\frac{g(C)}{R} \frac{\mathrm{d} R}{\mathrm{~d} t} \\
\frac{\partial n}{\partial t}+\frac{\partial}{\partial C}\left(n \frac{\mathrm{d} C}{\mathrm{~d} t}\right)=0
\end{gathered}
$$

with the boundary condition : $(n \mathrm{~d} C / \mathrm{d} t)\left(C_{\min }\right)=2(n \mathrm{~d} C / \mathrm{d} t)\left(C_{\max }\right)$.

\section{Self-similar solution.}

A self-similar solution of equations (7)-(9) can be found for which $R(t)=(\eta t)^{1 / 2}$. Then, from equation (7) the distribution function $n$ must be taken of the form $n=t^{1 / 4} N(C)$ where $N$ is normalized as :

$$
2 \pi \sqrt{\sqrt{\eta} \frac{\mathrm{Ca}}{b}}=\int_{C_{\min }}^{C_{\max }} \sqrt{g(C)} N(C) \mathrm{d} C .
$$

From equation (9), one obtains :

$$
\frac{\mathrm{d} C}{\mathrm{~d} t}=\frac{1}{t} \Gamma(Q / \eta, C)
$$

where

$$
\Gamma(Q / \eta, C)=\frac{1}{g^{\prime}(C)}\left(\frac{Q}{\pi \eta} C-\frac{g(C)}{2}\right)
$$

from which follows the equation for $N(C)$ :

$$
\frac{1}{4} N+\frac{\mathrm{d}}{\mathrm{d} C}(N \Gamma)=0 .
$$

This equation can easily be integrated to obtain

$$
N(C)=A \exp \int_{C}^{C_{\max }} \frac{1+4 \frac{\mathrm{d} \Gamma}{\mathrm{d} C}}{4 \Gamma} \mathrm{d} C^{\prime}
$$


Here, the factor $\eta$ appears as an eigenvalue which will be determined by the boundary condition

$$
N\left(C_{\min }\right) \Gamma\left(C_{\min }\right)=2 N\left(C_{\max }\right) \Gamma\left(C_{\max }\right) .
$$

The normalization constant $A$ is then determined by equation (10). As the condition of tipsplitting at constant outer fluid velocity determines $C_{\min }$ as a function of $C_{\max }, Q / \pi \eta$ is only a function of $C_{\max }$. As $C_{\max } \approx-\log \left(A_{1}\right)$, where $A_{1}$ is the relative amplitude of the noise present in the system [2], the self-similar distribution function and the associated physical quantities characterizing the growth of the effective interface are determined by the amplitude of noise in the Hele Shaw cell. To proceed further, we use an interpolation formula of the McLean and Saffman function $\lambda(C)=\left(\left(C-C_{0}\right)+A\right) /\left(2\left(C-C_{0}\right)+A\right)$ with $C_{0}=2.87$ and $A=50.8$ which reproduces within a few percent, their numerical data for values of $\lambda$ ranging between 1.0 and 0.6 [6]. In figure 2 plotted the factor $Q / \pi \eta$ is plotted as a function of $C_{\max }$. This factor characterizes the ratio between the inner volume outlined by the envelope and the total volume of displacing fluid injected, since $Q / \pi \eta=Q b t / \pi R^{2} b$. Figure 3 shows the normalized distribution function $N(C) /\left(2 \pi \eta^{1 / 4}(\mathrm{Ca} / b)^{1 / 2}\right)$ as a function of $C$ for $C_{\max }=10$.

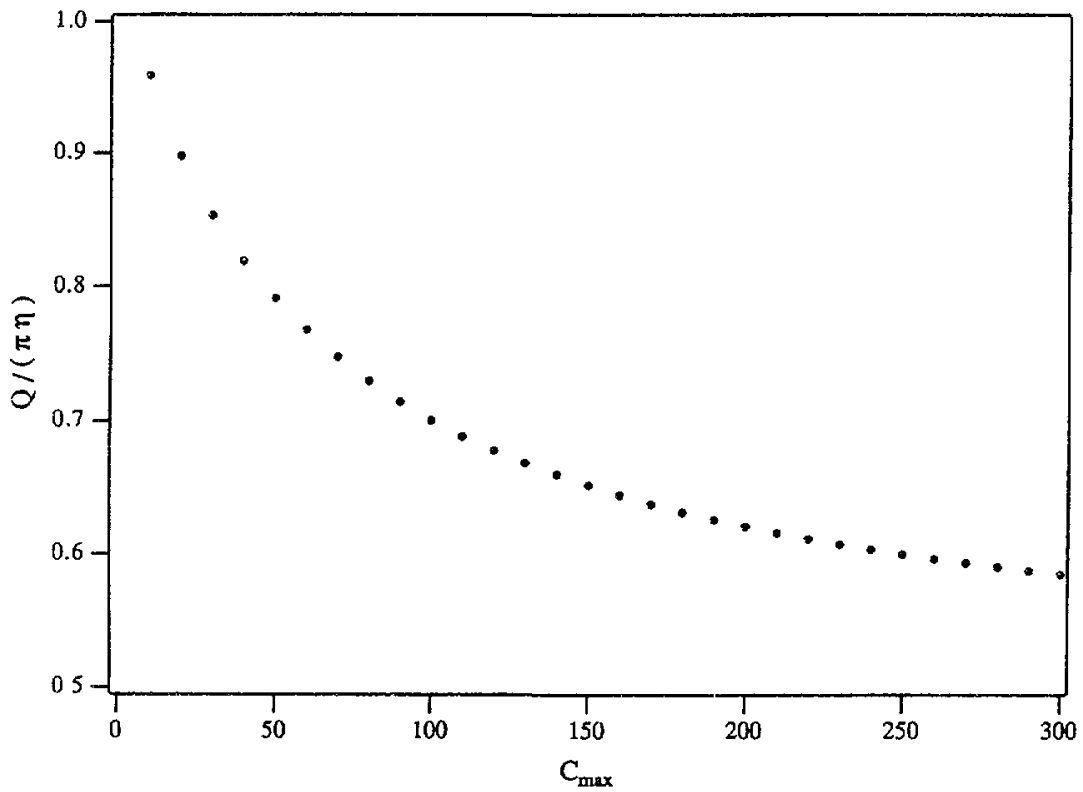

Fig. 2. - Volumetric ratio $Q / \pi \eta$ as a function of $C_{\max }$.

\section{Numerical integration.}

Numerical solutions of equations (7)-(9) for which $\lambda(C)$ is the McLean and Saffman function. The numerical values of the parameters are the same as in the Paterson experiment [1], $Q=9.3 \mathrm{~cm}^{2} / \mathrm{s}, b=0.15 \mathrm{~cm}$ and $\mathrm{Ca}=0.25$. Figure $4 \mathrm{a}$ shows the distribution function as a function of $C$ for different times. One has divided the distribution function by $t^{1 / 4}$, scaling 


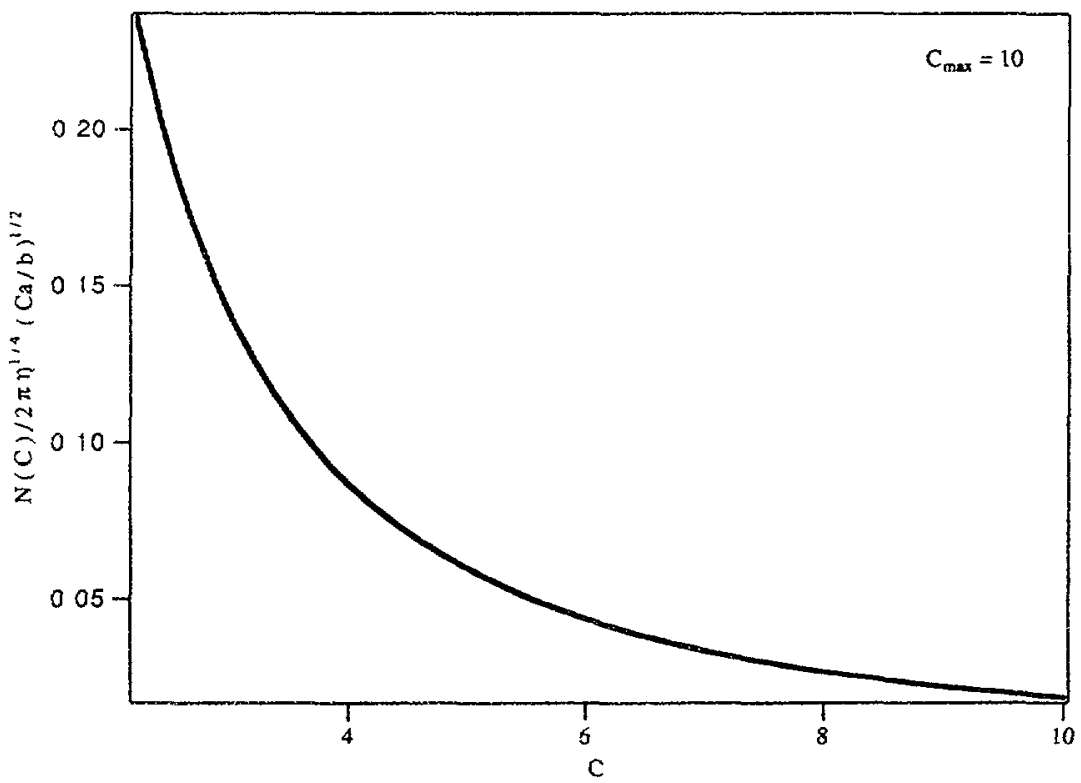

Fig. 3. - Distribution function $N(C) / 2 \pi \eta^{1 / 4}(\mathrm{Ca} / b)^{1 / 2}$ as a function of $C$.
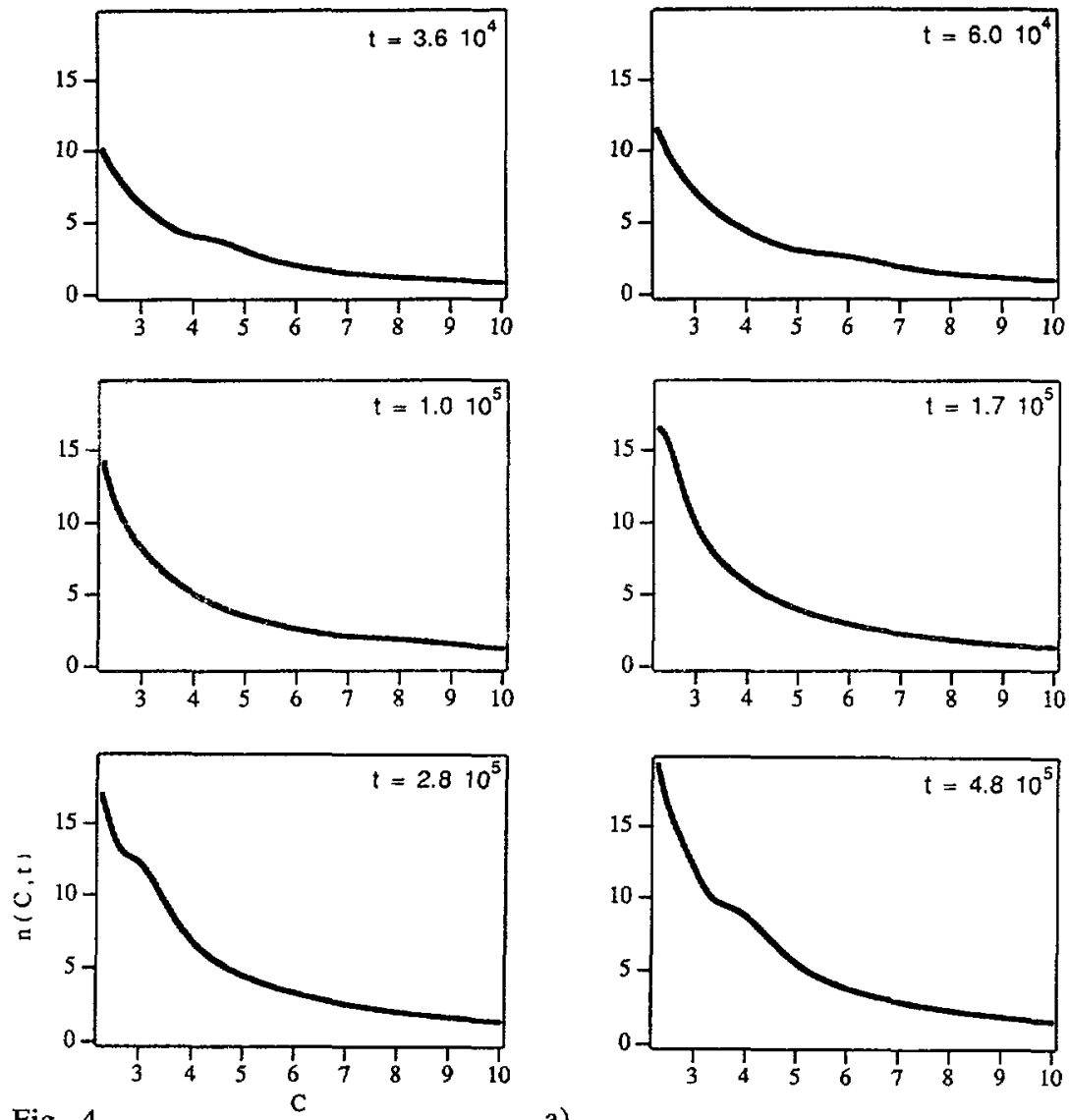

Fig. 4.

a) 

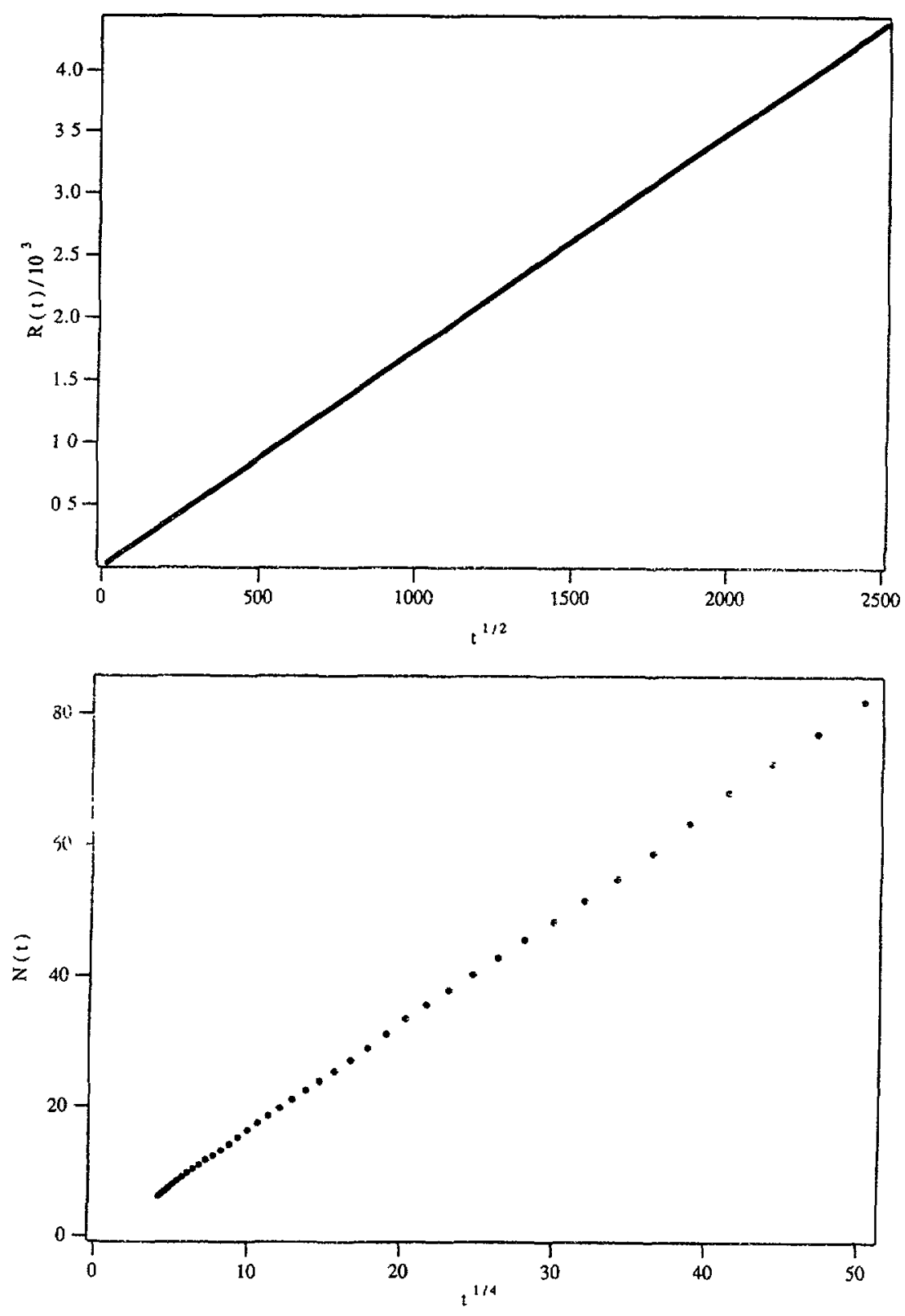

b)

Fig. 4. - a) Distribution function $n(C)$ at different times (time unit : seconds) starting from an initial condition close to the self-similar solution. b) Corresponding evolution of the mean radius of the germ $R(t)$ (length unit : $\mathrm{cm}$ ) and the total number of cells $N(t)$.

expected for the self-similar solution. The initial condition is the self-similar solution determined above on which a little bump in the $C$ space is added. When the time elapses this bump evolves towards larger $C$ values. Then tip splitting occurs and the bump is redistributed towards smaller values. In figure $4 \mathrm{~b}$ are shown the graphs of $R(t)$ and $N(t)$ the total number of 
cells. There is no clear deviation from the self-similar values. In figure 5a the integration of the distribution function starts from an initial Gaussian function. As previously, the bump moves towards larger $C$ values due to the increase of the mean radius of the germ. Then tip-splitting occurs and the bump is redistributed towards smaller values of $C$. The corresponding values of $R(t)$ and $N(t)$ are shown in figure $5 \mathrm{~b}$.
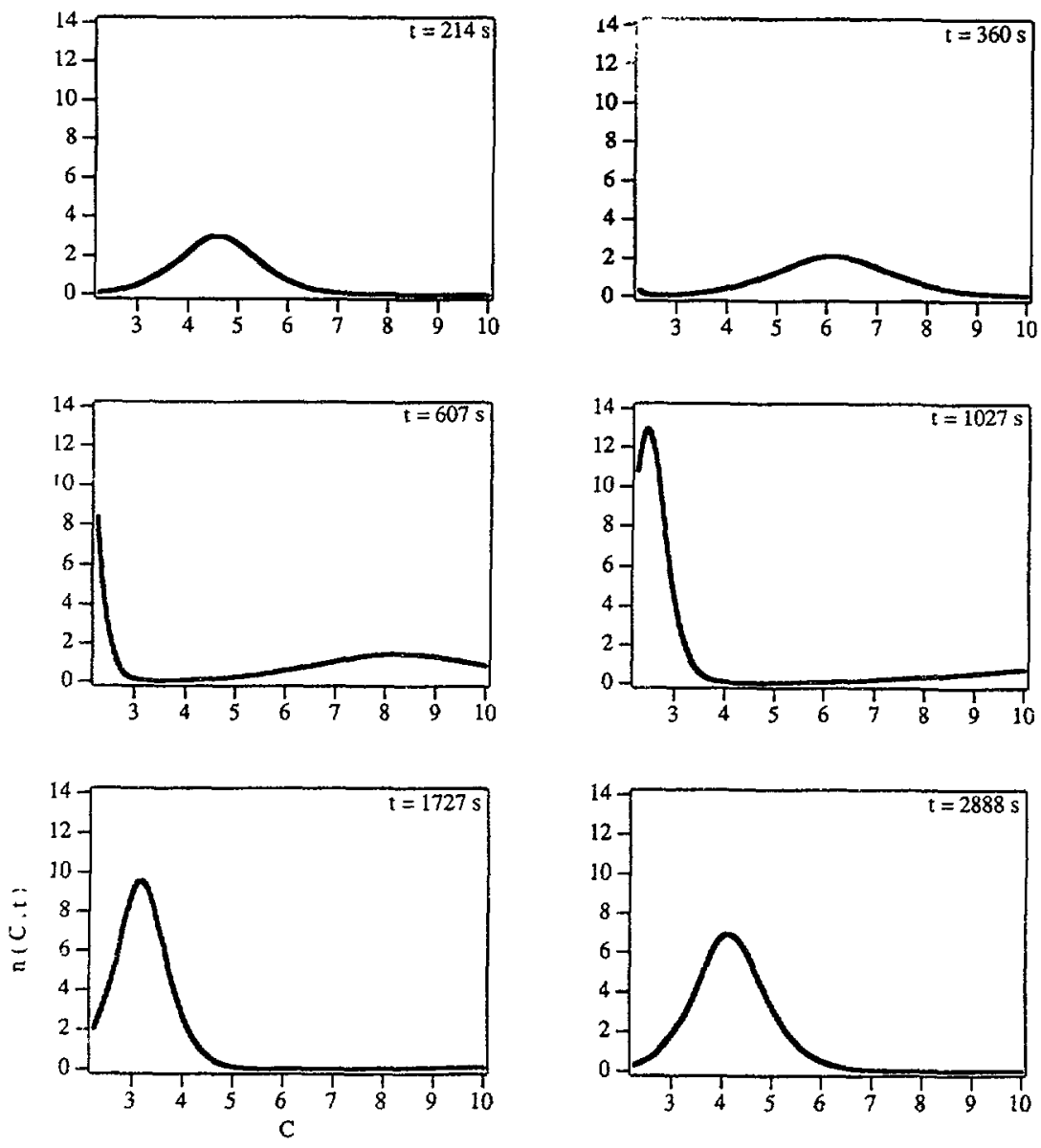

a)

Fig. 5. - a) Distribution function $n(C)$ at different times starting from a Gaussian initial condition. b) Corresponding evolution of the mean radius of the germ $R(t)$ and the total number of cells $N(t)$. 

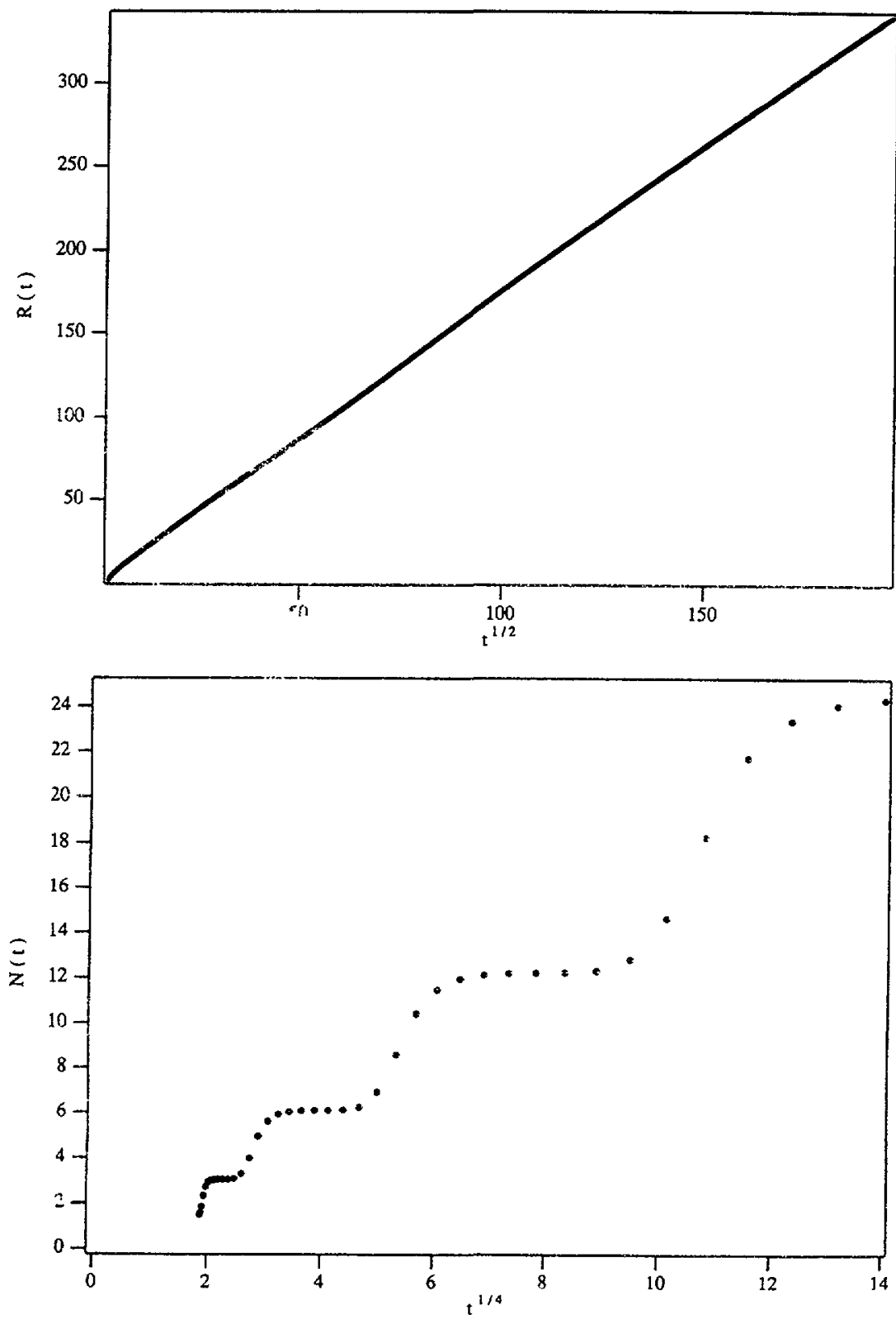

b)

Fig. 5 (continued).

\section{Conclusion.}

We have used a statistical method to describe an unsteady radial fingering pattern expanding in a Hele Shaw cell. Without taking into account the cell death process, we determine a complete set of equations for the growth of the envelope of the pattern which couples effective radius, individual growth rate for a unit cell, and the distribution function in the cell size space. A self 
similar solution of these equations can be determined, for which the time dependence of radius and distribution function are respectively in $t^{1 / 2}$ and $t^{1 / 4}$ The prefactor of these power laws is a function of the amplitude of the noise present in the Hele Shaw cell.

It is interesting to notice that the self similar solution characterizes completely the microstructure and the dynamic variables of the growth. This is not the case if patterns with uniform wavelength are considered. As the wavelength is arbitrary, arbitrary growth velocity is allowed too. But if one starts from reasonable initial conditions, one can expect that the microstructure evolves towards the self-similar state growing in a unique fashion. We expect that such an approach can be useful too, for the directional solidification configuration where the problem of wavelength selection is not yet understood.

\section{Acknowledgments.}

The authors are grateful to E. Brener, P. Oswald. This work was performed with the fundings of CNES-PIRMAT.

\section{References}

[1] Paterson L., J. Fluid Mech. 113 (1981) 513.

[2] PelCE P., Dynamics of Curved Fronts (Academic Press, 1988).

[3] Datye V., Mathur R. and Langer J. S., J. Stat. Phys. 29 (1982) 1.

[4] Lifshitz I. and Slyozov V., J. Phys. Chem. Solids 19 (1961) 35.

[5] Karma A. and Pelce P., Phys. Rev. A 41 (1990) 6741.

[6] MCLean J. W. and SafFMan P. G., J. Fluid Mech. 102 (1981) 455.

[7] Ben Amar M., Phys. Rev. A 44 (1991) 3673.

[8] Vanden-Broeck J. M., Phys. Fluids 26 (1983) 191. 